Article

\title{
Journals in Aging, Geriatrics, and Gerontology: A Survey
}

\author{
Robert J. Wolff ${ }^{1}$ and Hannah L. Bowser ${ }^{2}$ \\ $1 \quad$ Professor of Science and Health Science, South University; rwolff@ southuniversity.edu \\ $2 \quad$ Virtual Services Librarian, New Hanover County Public Library; hbowser@ nhcgov.com
}

\begin{abstract}
Aging, geriatrics, gerontology, and related areas are important areas of research as the population of older people increases in relationship to the total population. Researchers in these fields would benefit from guidance regarding sources for publishing and finding relevant scholarly journals and articles. Multiple database sites of journals were searched to provide a list of relevant publications. This list was expanded via perusal of published citation lists and searches in general search engines. A total of 243 journals were identified and examined. Of those journals, 198 journals are currently publishing and 45 journals have ceased publication. In terms of publication medium, $39 \%$ of the currently publishing journals are online-only, $4 \%$ are print-only and 59\% of the journals publish both online and in print. Journals with aging in the title represent $36 \%$, geriatrics $30 \%$ and gerontology $23 \%$. Less than $10 \%$ have been identified as predatory journals. An expected increase in journals in the broad field of aging is indicated by the $49 \%$ of listed titles beginning in 2000 or later. This recent increase in available journals provides a need for the information listed in this paper.
\end{abstract}

Keywords: Aging; Ageing; Geriatric; Gerontology; Medical journals; Medical publishing; Predatory journals; dementia; nursing research.

\section{Introduction}

Not only is the population of elderly people rapidly increasing as a demographic, so is the number and variety of journals that publish research in the areas of aging, geriatrics and gerontology. Researchers are now finding it difficult to determine what journals they can find, which are appropriate, and how to easily access their websites.

While there are some listings of journals that can be found using search engines, none of these are very complete, nor do they provide easy access to the journals. Having a greater knowledge of, and access to, the wide variety of publishing options can make it easier for researchers and librarians to advance their professional endeavors.

The purposes of this paper include the following:

1. To document the variety of journals available in the professional areas of aging, geriatrics and gerontology.

2. To provide a list of journals that is as comprehensive as possible. 
medRxiv preprint doi: https://doi.org/10.1101/19012278; this version posted November 22, 2019. The copyright holder for this preprint (which was not certified by peer review) is the author/funder, who has granted medRxiv a license to display the preprint in perpetuity.

It is made available under a CC-BY-ND 4.0 International license .

3. To help researchers and graduate students find appropriate journals in which to publish their work.

4. To open up options for other sources for publication of their research and commentaries.

5. To examine various issues in the diversity of journals in these areas.

6. To provide some warning about journals that are possibly predatory and therefore need to be avoided by serious researchers, and whose articles may be of poor quality.

\section{Materials and Methods}

The Scimago Journal website [1], the Clarivate Analytics (Web of Science) [2], JenAge Information Center [3], and PubMed (NLM) [4] were used to access lists of journals related to aging. To these we added journals found in citation listings, on publisher websites, and from internet searches. Listings of the A-Z journal titles found in the South University library system were also searched, using keywords such as "age," "aging," "gerontology," etc.

The website of each journal was searched and checked for publication status and information about online and open access features, and if available, the article processing charges were determined.

Our listing of predatory journals is based on Beall's List of Predatory Journals and Publishers, which can now be accessed in several internet sites. We used an anonymous Weebly blog [5] that was last updated 4 July 2018.

\subsection{A Note on Predatory Publishing}

Predatory journals are defined by the authors as journals that seek submissions at a high price but generally publish low quality information and often under false pretenses. Predatory journals are included in our list for three reasons. First, because they exist, it is important to list and identify them. Second, they are included so their listing can be found and avoided, by those seeking a reputable journal in which to publish. Third, we want them identified as predatory journals so that their status is clearly noted and so their published articles may be carefully analyzed as they are often of poor quality.

The problems of predatory journals and publishers have been clearly documented, though not without conflict. Warnings about predatory journals have been sounded as a threat to and an undermining of confidence in scholarly research [6-8]. Criteria are available for recognition of legitimate or predatory journals [9-11]. However, it is worth noting that the open access model of publishing does not automatically denote a predatory journal, nor are all predatory journals open access publishers and journals. Although the authors used Beall's List as a convenient listing of predatory journals, it is important to note that this list has its critics $[12,13]$ and that the best methods of recognizing a predatory publisher or journal are through each author's own research $[9,10]$. 


\section{Results}

The number of currently publishing journals in the broad fields of aging, geriatrics and gerontology that is we focused on is 198 , and we found 45 titles that have ceased publication. Table 1 provides basic information about the content of the journals, and the use of common terms or prefixes in journal titles. Age or Aging, with the spelling variant of Ageing, and the Portuguese Envelhecimento, were the most common descriptors used in journal titles at 70 (36\%). This was followed by Geri- or Geriatric, or the Dutch Ouderengeneeskunde, with 59 $(30 \%)$ uses.

The journals were categorized as Print (only), Online, or Both. Only 7 of the currently publishing journals were print only (4\%), while 5 of the 45 journals that have ceased publication (11\%) were print only, which may represent the slow demise of print journals.

Of the currently publishing journals, 76 (39\%) were online only, and 115 (59\%) were published both online and in print form. One journal was considered to be a print publication, with only society members having access to the online version.

Journals with 19 or less volumes published as of September 2018 numbered 98 (50\%), indicating the rapid expansion of journals since 2000. Of the journals that have ceased publication, 29 (65\%) began in 2000 or after, and if 1998-99 are included, the number jumps to $34(76 \%)$, indicating the difficulty of journal start-ups to maintain publication during the move to open access publishing.

Nineteen of the currently publishing journals were identified as potentially predatory journals or publishers, just less than $10 \%$ of all available journals in the broad area of aging. We did not find any hijacked journals (where websites or branding were coopted by a predatory publisher), but the similarity of some journal names may be a form of hijacking by attempting to create confusion.

The complete list of journals that are currently being published may be found in Table 2 and the complete list of journals that have ceased publication is in Table 3.

Table 1. Journal areas of content (Currently publishing)

\begin{tabular}{ccc}
\hline Journal Titles $^{1}$ & Number & Percent (of 195) \\
Age, Aging, Ageing, Envelhecimento & 70 & $36 \% \%^{2}$ \\
Gero-, Gerontology & 45 & $23 \%$ \\
Geri-, Geriatric, Ouderengeneeskunde & 59 & $30 \%$ \\
\hline
\end{tabular}


Alzheimers, Dementia

Soc-, Social

Neuro-, Mental

Psych-

Nurs-, Nursing

Elder, Elderly, Older

Parkinson-
21

8

12

14

7

7

7
$11 \%$

$4 \%$

$6 \%$

$8 \%$

$4 \%$

$4 \%$

$4 \%$

${ }^{1}$ Some titles included more than one of the words or prefixes.

${ }^{2}$ Percentages were rounded up to the whole number.

\section{Discussion}

The list provides clear indication that the print only journal is on its way out, with 7 currently published journals in print only, compared to 115 journals in both print and online formats, and 76 online only journals. We expect to see many of the journals still in print or both formats to move to online only within the next few years.

We are requesting that journal editors and publishers send updated information to the senior author to help improve the list and information about the journals. Missing journals need to be added to the list after they are identified. It is our expectation that updated versions of this paper may be published in a preprint form in the future. This can more easily be updated on a regular basis, or made available in some other format.

Of interest is the apparently low number of journals focusing on nursing in the field of gerontology. Only 7 of the 195 (4\%) currently published journals specifically refer to nursing, a low number as it seems that most nurses do work with aging patients. Those in fields of sociology and social work as well as psychology seem under-represented. Another area of concern is the lack of journals publishing research focusing on caregiving, with the only journal we found in this area having ceased publication. The area of caregiving currently has an emphasis on problems of the health of caregivers and the problems associated with poor (even abusive) quality of care for the aged. This is also a field often staffed by a nonacademic populace who would not have easy access to the wealth of information potentially available on current gerontology and caregiving research. 
It is apparent from a librarian's point of view that it is difficult for the average researcher to find and compile a complete list of potential publishing outlets, and it would be helpful to the scholarly community to have an easily searchable database, preferably not behind a firewall. This could increase the diversity of journal articles being published and help researchers find more appropriate choices for article submission, as well as increasing the diversity of authors who are submitting works for publication.

With the rise in predatory publishing, we consider that having only $10 \%$ of the journals a low number, though there should be careful evaluation of all journals including newly launched titles. Additional journal titles are expected to be found as many of the predatory journals/publishers are not found in searches. More research could be done in specific scholarly fields as to the perceptions of predatory journals as well as how the rise of the open access movement has affected fields of research for better or for worse.

\section{Conclusions}

This paper provides a current update on journals being published in the broad disciplines of aging, geriatrics and gerontology and providing a broad view of research being published in the area. It is expected that this will be a useful resource to researchers who are considering publishing their research, to those trying to make sure they find all relevant articles in their searches, and for those looking at the discipline of aging, geriatrics and gerontology as a whole.

The data provide a view of the wide areas covered within the broad disciplines involved in aging, and the increasing numbers of journals does indicate both an increased focus on aging as well as the dynamic nature of the expanding aspects of aging. While many general journals publish articles on areas of aging, these specific journals are important as a way to view the activity and interests in these fields. While the increase in journal numbers and types is not a direct concern, it is critical that the field note the quality of journals, and that efforts are made to help other researchers avoid predatory journals where there are high costs but the quality of the reviews and papers are very low. This not only hurts the field and is a waste of money, but also undermines the research and medical communities and confidence in them.

This compiled list of data will hopefully be a jumping off point for other fields of research to compile lists and bibliographies of available journals for publication and their potential value or risk. It will be especially helpful to students and new researchers in the field as a starting point for their projects and careers.

Author Contributions: Conceptualization, R.W.; methodology, R.W.; investigation, H.B.; data curation, H.B.; writing — original draft preparation, H.B. and R.W.; writing-review and editing, H.B. and R.W.; visualization, H.B.; supervision, R.W.; project administration, R.W.

Funding: This research received no external funding.

Acknowledgments: Thanks for support and editing to Donna Wolff.

Conflicts of Interest: The authors declare no conflict of interest. 
medRxiv preprint doi: https://doi.org/10.1101/19012278; this version posted November 22, 2019. The copyright holder for this preprint (which was not certified by peer review) is the author/funder, who has granted medRxiv a license to display the preprint in perpetuity.

It is made available under a CC-BY-ND 4.0 International license.

\begin{tabular}{|c|c|c|c|c|c|c|c|}
\hline \multicolumn{8}{|c|}{ TABLE 2} \\
\hline \multicolumn{8}{|c|}{ JOURNALS CURRENTLY IN PUBLICATION } \\
\hline \# & $\begin{array}{l}\text { Name of Journal /ISSN } \\
\text { (Print; Online) }\end{array}$ & Publisher/Society & $\begin{array}{l}\text { Number of } \\
\text { Volumes }\end{array}$ & $\begin{array}{l}\text { Print or } \\
\text { Online? }\end{array}$ & $\begin{array}{l}\text { Open } \\
\text { Access? } \\
\text { APC }(\$)\end{array}$ & $\begin{array}{l}\text { Impact Factor/ } \\
\text { Scimago } \\
\text { Rank }^{*}\end{array}$ & $\begin{array}{l}\text { Predatory } \\
\text { Publisher? }\end{array}$ \\
\hline 1. & $\begin{array}{l}\text { Activities, Adaptation \& } \\
\text { Aging } \\
0192-4788 ; 1544-4368\end{array}$ & $\begin{array}{l}\text { Taylor \& Francis } \\
\text { Online }\end{array}$ & 41 & Both & Optional & 0.44 & \\
\hline 2. & $\begin{array}{l}\text { Advances in Aging } \\
\frac{\text { Research }}{2169-0499 ;} ; 2169-0502\end{array}$ & $\begin{array}{l}\text { Scientific } \\
\text { Research } \\
\text { Publishing, Inc. }\end{array}$ & 7 & Online & $\begin{array}{l}\text { Yes; APC } \\
\$ 599\end{array}$ & & $\begin{array}{l}\text { Yes (Beall's } \\
\text { List) }\end{array}$ \\
\hline 3. & $\begin{array}{l}\frac{\text { Advances in Alzheimer's }}{\text { Disease }} \\
2169-2459 ; 2169-2467\end{array}$ & $\begin{array}{l}\text { Scientific } \\
\text { Research } \\
\text { Publishing, Inc. }\end{array}$ & 7 & Online & $\begin{array}{l}\text { Yes; APC } \\
\$ 599\end{array}$ & & $\begin{array}{l}\text { Yes (Beall's } \\
\text { List) }\end{array}$ \\
\hline 4. & $\frac{\text { Advances in Gerontology }}{2079-0570 ; 2079-0589}$ & Springer Nature & 7 & Both & No & 0.32 & \\
\hline 5. & $\begin{array}{l}\text { Advances in Life Course } \\
\frac{\text { Research }}{1569-4909 ; 1879-} \\
6974 / 1040-2608\end{array}$ & Elsevier & 37 & Both & $\begin{array}{l}\text { Optional; } \\
\text { APC } \$ 1100\end{array}$ & 1.200 & \\
\hline 6. & $\begin{array}{l}\text { Advances in Parkinson's } \\
\frac{\text { Disease }}{2169-9712 ; 2169-9720}\end{array}$ & $\begin{array}{l}\text { Scientific } \\
\text { Research } \\
\text { Publishing, Inc. }\end{array}$ & 7 & Online & $\begin{array}{l}\text { Yes; APC } \\
\$ 499\end{array}$ & & $\begin{array}{l}\text { Yes (Beall's } \\
\text { List) }\end{array}$ \\
\hline 7. & $\begin{array}{l}\text { Age and Ageing } \\
0002-0729 ; 1468-2834\end{array}$ & Oxford Academic & 46 & Both & $\begin{array}{l}\text { Optional; } \\
\text { APC } \$ 3400\end{array}$ & 4.282 & \\
\hline 8. & $\begin{array}{l}\frac{\text { Age, Culture, }}{\text { Humanities }} \\
\frac{2375-8856 ; 2373-5481}{}\end{array}$ & Athenaeum Press & 3 & Both & No & & \\
\hline 9. & $\frac{\text { Ageing International }}{0163-5158 ; 1936-606 X}$ & Springer Nature & 42 & Both & $\begin{array}{l}\text { Optional; } \\
\text { APC } \$ 3000\end{array}$ & 0.39 & \\
\hline 10. & $\begin{array}{l}\frac{\text { Ageing Research }}{\text { Reviews }} \\
1568-1637\end{array}$ & Elsevier & 46 & Both & $\begin{array}{l}\text { Optional; } \\
\text { APC } \$ 3000\end{array}$ & 7.452 & \\
\hline 11. & $\begin{array}{l}\text { Ageing \& Society } \\
\text { 0144-686X; 1469-1779 }\end{array}$ & $\begin{array}{l}\text { Cambridge } \\
\text { University Press }\end{array}$ & 37 & Both & $\begin{array}{l}\text { Optional; } \\
\text { APC } \$ 2835\end{array}$ & 1.386 & \\
\hline 12. & $\begin{array}{l}\text { Aging } \\
\underline{1945-4589}\end{array}$ & $\begin{array}{l}\text { Impact Journals, } \\
\text { LLC }\end{array}$ & 9 & Online & $\begin{array}{l}\text { Yes; APC } \\
\$ 2900\end{array}$ & 4.867 & $\begin{array}{l}\text { Yes (Beall's } \\
\text { List) }\end{array}$ \\
\hline 13. & $\frac{\text { Aging Cell }}{1474-9726}$ & $\begin{array}{l}\text { John Wiley \& } \\
\text { Sons }\end{array}$ & 17 & Online & $\begin{array}{l}\text { Yes; APC } \\
\$ 2900\end{array}$ & 6.714 & \\
\hline 14. & $\begin{array}{l}\frac{\text { Aging Clinical and }}{\text { Experimental Research }} \\
\frac{1720-8319}{}\end{array}$ & Springer Nature & 30 & Online & $\begin{array}{l}\text { Optional; } \\
\text { APC } \$ 3000\end{array}$ & 1.394 & \\
\hline 15. & $\frac{\text { Aging and Disease }}{2152-5250}$ & $\begin{array}{l}\text { Beijing Magtech } \\
\text { Co. Ltd }\end{array}$ & 8 & Online & $\begin{array}{l}\text { Yes; APC } \\
\$ 600-2000\end{array}$ & 5.058 & \\
\hline 16. & $\frac{\text { Aging Male, The }}{1368-5538 ; 1473-0790}$ & $\begin{array}{l}\text { Taylor \& Francis } \\
\text { Online }\end{array}$ & 20 & Both & $\begin{array}{l}\text { Yes } \\
\text { (Optional) }\end{array}$ & 2.108 & \\
\hline 17. & $\frac{\text { Aging \& Mental Health }}{1360-7863 ; 1364-6915}$ & $\begin{array}{l}\text { Taylor \& Francis } \\
\text { Online }\end{array}$ & 21 & Both & $\begin{array}{l}\text { Yes } \\
\text { (Optional); } \\
\text { APC } \$ 2950\end{array}$ & 2.658 & \\
\hline 18. & $\begin{array}{l}\text { Aging, } \\
\text { Neuropsychology, and } \\
\text { Cognition }\end{array}$ & $\begin{array}{l}\text { Taylor \& Francis } \\
\text { Online }\end{array}$ & 25 & Both & $\begin{array}{l}\text { Yes } \\
\text { (Optional); } \\
\text { APC } \$ 2950\end{array}$ & 1.763 & \\
\hline
\end{tabular}


medRxiv preprint doi: https://doi.org/10.1101/19012278; this version posted November 22, 2019. The copyright holder for this preprint (which was not certified by peer review) is the author/funder, who has granted medRxiv a license to display the preprint in perpetuity.

It is made available under a CC-BY-ND 4.0 International license.

\begin{tabular}{|c|c|c|c|c|c|c|c|}
\hline & 1382-5585; 1744-4128 & & & & & & \\
\hline 19. & $\frac{\text { Aging-US }}{1945-4589}$ & Impact Journals & 10 & Online & $\begin{array}{l}\text { Yes; APC } \\
\$ 2900\end{array}$ & 4.867 & $\begin{array}{l}\text { Yes (Beall's } \\
\text { List) }\end{array}$ \\
\hline 20. & AGS Newsletter & $\begin{array}{l}\text { American } \\
\text { Geriatrics Society }\end{array}$ & 48 & Online & Yes & & \\
\hline 21. & $\begin{array}{l}\frac{\text { Alzheimer Disease and }}{\text { Associated Disorders }} \\
\text { 0893-0341; 1546-4156 }\end{array}$ & Wolters Kluwer & 32 & Both & $\begin{array}{l}\text { Optional; } \\
\text { APC } \$ 2800\end{array}$ & 2.395 & \\
\hline 22. & $\begin{array}{l}\frac{\text { Alzheimer's and }}{\text { Dementia }} \\
1552-5260\end{array}$ & Elsevier & 13 & Both & $\begin{array}{l}\text { Yes } \\
\text { (Optional); } \\
\text { APC } \$ 3000\end{array}$ & 9.478 & \\
\hline 23. & $\begin{array}{l}\text { Alzheimer's and } \\
\text { Dementia: Diagnosis, } \\
\text { Assessment \& Disease } \\
\text { Monitoring } \\
2352-8729\end{array}$ & Elsevier & 10 & Online & $\begin{array}{l}\text { Yes; APC } \\
\$ 2000\end{array}$ & 1.796 & \\
\hline 24. & $\begin{array}{l}\text { Alzheimer's and } \\
\text { Dementia: Translational } \\
\text { Research \& Clinical } \\
\text { Interventions } \\
2352-8737\end{array}$ & Elsevier & 3 & Online & $\begin{array}{l}\text { Yes; APC } \\
\$ 2000\end{array}$ & 1.108 & \\
\hline 25. & $\begin{array}{l}\text { Alzheimer's and } \\
\text { Parkinsonism: Research } \\
\text { \& Therapy }\end{array}$ & SM Online & 1 & Online & $\begin{array}{l}\text { Yes; APC } \\
\text { not given }\end{array}$ & & $\begin{array}{l}\text { Yes (Beall's } \\
\text { List) }\end{array}$ \\
\hline 26. & $\begin{array}{l}\text { Alzheimer's Research \& } \\
\text { Therapy } \\
\text { 1758-9193 }\end{array}$ & BioMed Central & 9 & Online & $\begin{array}{l}\text { Yes; APC } \\
\$ 2175\end{array}$ & 6.154 & \\
\hline 27. & $\begin{array}{l}\frac{\text { American Journal of }}{\text { Alzheimer's Disease and }} \\
\frac{\text { Other Dementias }}{1533-3175 ; 1938-2731}\end{array}$ & $\begin{array}{l}\text { SAGE } \\
\text { Publications }\end{array}$ & 32 & Both & No & 1.864 & \\
\hline 28. & $\begin{array}{l}\frac{\text { American Journal of }}{\text { Geriatric Psychiatry, The }} \\
\text { 1064-7481 }\end{array}$ & Elsevier & 25 & Both & $\begin{array}{l}\text { Yes } \\
\text { (Optional); } \\
\text { APC } \$ 3300\end{array}$ & 2.868 & \\
\hline 29. & $\begin{array}{l}\text { Anales en Gerontología } \\
\text { (Annals in Gerontology) } \\
1659-0813 ; 2215-4647\end{array}$ & $\begin{array}{l}\text { University of } \\
\text { Costa Rica }\end{array}$ & 8 & Online & Yes & & \\
\hline 30. & $\begin{array}{l}\text { Annals of Geriatric } \\
\text { Medicine and Research } \\
\text { (formerly Journal of the } \\
\text { Korean Geriatrics } \\
\frac{\text { Society) }}{2508-4798 ; 2508-4909}\end{array}$ & $\begin{array}{l}\text { The Korean } \\
\text { Geriatrics Society }\end{array}$ & 22 & Both & Yes & & \\
\hline 31. & $\begin{array}{l}\text { Annals of Long-Term } \\
\text { Care } \\
1524-7929\end{array}$ & $\begin{array}{l}\text { HMP } \\
\text { Communications }\end{array}$ & 25 & Both & Yes & 0.14 & \\
\hline 32. & $\begin{array}{l}\frac{\text { Annual Review of }}{\text { Gerontology and }} \\
\frac{\text { Geriatrics }}{\text { 0198-8794; 1944-4036 }}\end{array}$ & $\begin{array}{l}\text { Springer } \\
\text { Publishing } \\
\text { Company }\end{array}$ & 37 & Both & No & 0.222 & \\
\hline 33. & $\begin{array}{l}\text { Anthropology \& Aging } \\
\text { (formerly Anthropology }\end{array}$ & $\begin{array}{l}\text { University of } \\
\text { Pittsburgh }\end{array}$ & 38 & Online & $\begin{array}{l}\text { Yes; AAGE } \\
\text { Membership }\end{array}$ & & \\
\hline
\end{tabular}


medRxiv preprint doi: https://doi.org/10.1101/19012278; this version posted November 22, 2019. The copyright holder for this preprint (which was not certified by peer review) is the author/funder, who has granted medRxiv a license to display the preprint in perpetuity.

It is made available under a CC-BY-ND 4.0 International license.

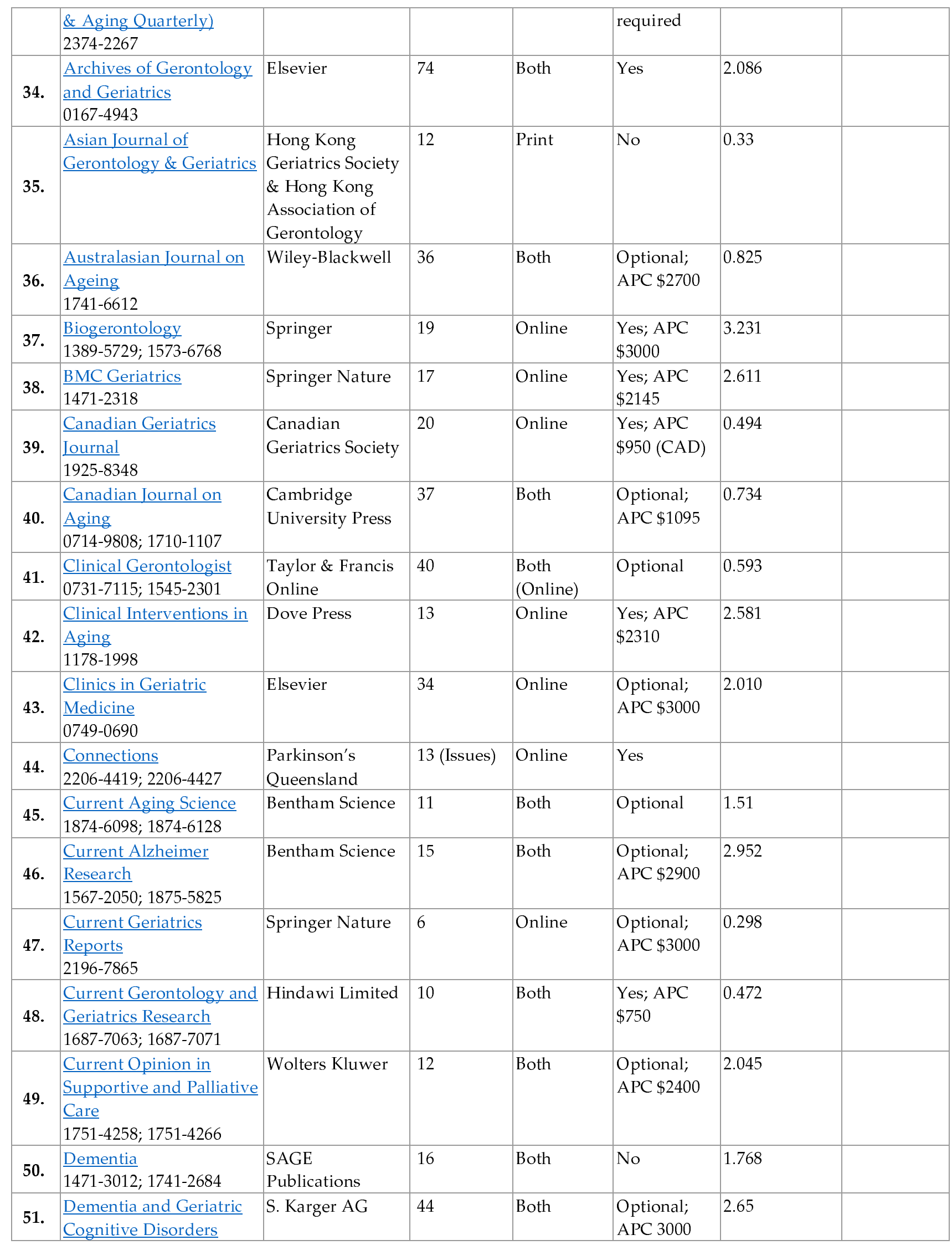


medRxiv preprint doi: https://doi.org/10.1101/19012278; this version posted November 22, 2019 . The copyright holder for this preprint (which was not certified by peer review) is the author/funder, who has granted medRxiv a license to display the preprint in perpetuity.

It is made available under a CC-BY-ND 4.0 International license .

\begin{tabular}{|c|c|c|c|c|c|c|}
\hline & 1420-8008; 1421-9824 & & & & (CHF) & \\
\hline 52. & $\begin{array}{l}\text { Dementia and Geriatric } \\
\text { Cognitive Disorders } \\
\text { Extra } \\
\text { 1664-5464 }\end{array}$ & S. Karger AG & 7 & Online & $\begin{array}{l}\text { Yes; APC } \\
1800 \text { (CHF) }\end{array}$ & 0.632 \\
\hline 53. & $\begin{array}{l}\frac{\text { Dementia and }}{\text { Neurocognitive }} \\
\frac{\text { Disorders }}{1738-1495 ; 2384-0757}\end{array}$ & $\begin{array}{l}\text { Korean Dementia } \\
\text { Association }\end{array}$ & 17 & Both & Yes & \\
\hline 54. & $\begin{array}{l}\frac{\text { Dementia e }}{\text { Neuropsychologia }} \\
1980-5764\end{array}$ & $\begin{array}{l}\text { Associação } \\
\text { Neurologia } \\
\text { Cognitiva e do } \\
\text { Comportamento }\end{array}$ & 12 & Online & No & 0.44 \\
\hline 55. & $\begin{array}{l}\text { Drugs and Aging } \\
\text { 1170-229X; 1179-1969 }\end{array}$ & Springer Nature & 34 & Both & $\begin{array}{l}\text { Optional; } \\
\text { APC } \$ 3000\end{array}$ & 2.769 \\
\hline 56. & $\begin{array}{l}\text { Educational Gerontology } \\
0360-1277 ; 1521-0472\end{array}$ & $\begin{array}{l}\text { Taylor \& Francis } \\
\text { Online }\end{array}$ & 44 & Both & $\begin{array}{l}\text { Optional; } \\
\text { APC } \$ 2950\end{array}$ & 0.674 \\
\hline 57. & $\frac{\text { Elderly Health Journal }}{2423-6179}$ & $\begin{array}{l}\text { Shahid Sadoughi } \\
\text { University of } \\
\text { Medical Sciences } \\
\text { and Health } \\
\text { Services }\end{array}$ & 4 & Online & Yes & \\
\hline 58. & $\begin{array}{l}\frac{\text { European Geriatric }}{\text { Medicine }} \\
1878-7657\end{array}$ & $\begin{array}{l}\text { Springer Nature } \\
\text { (formerly } \\
\text { Elsevier) }\end{array}$ & 8 & Online & $\begin{array}{l}\text { Optional; } \\
\text { APC } \$ 3000\end{array}$ & 1.336 \\
\hline 59. & $\begin{array}{l}\text { European Journal of } \\
\text { Ageing } \\
\text { 1613-9372; 1613-9380 }\end{array}$ & Springer Nature & 15 & Both & Unknown & 1.177 \\
\hline 60. & $\begin{array}{l}\frac{\text { European Review of }}{\text { Aging and Physical }} \\
\frac{\text { Activity }}{1861-6909}\end{array}$ & Springer Nature & 14 & Online & $\begin{array}{l}\text { Yes; APC } \\
\$ 2145\end{array}$ & 2.154 \\
\hline 61. & $\begin{array}{l}\text { Experimental Aging } \\
\frac{\text { Research }}{\text { 0361-073X; 1096-4657 }}\end{array}$ & $\begin{array}{l}\text { Taylor \& Francis } \\
\text { Online }\end{array}$ & 43 & Both & Optional & 1.345 \\
\hline 62. & $\begin{array}{l}\frac{\text { Experimental }}{\text { Gerontology }} \\
0531-5565\end{array}$ & Elsevier & 101 & Both & $\begin{array}{l}\text { Optional; } \\
\text { APC } \$ 2750\end{array}$ & 3.340 \\
\hline 63. & $\begin{array}{l}\text { Focus on Healthy Aging } \\
\text { (Mount Sinai School of } \\
\text { Medicine) }\end{array}$ & $\begin{array}{l}\text { Belvoir Media } \\
\text { Group LLC }\end{array}$ & 20 & Both & No & \\
\hline 64. & $\begin{array}{l}\text { Frontiers in Aging } \\
\frac{\text { Neuroscience }}{1663-4365}\end{array}$ & $\begin{array}{l}\text { Frontiers Media } \\
\text { SA }\end{array}$ & $\begin{array}{l}1690 \\
\text { articles }\end{array}$ & Online & $\begin{array}{l}\text { Yes; APC } \\
\$ 2950\end{array}$ & 1.638 \\
\hline 65. & $\frac{\text { Generations }}{0738-7806}$ & $\begin{array}{l}\text { American Society } \\
\text { on Aging }\end{array}$ & 42 & $\begin{array}{l}\text { Print } \\
\text { (Online for } \\
\text { members) }\end{array}$ & No & 0.31 \\
\hline 66. & $\begin{array}{l}\frac{\text { Gériatrie et Psychologie }}{\text { Neuropsychiatrie du }} \\
\frac{\text { Vieillissement }}{2115-8789 ; 2115-7863}\end{array}$ & $\begin{array}{l}\text { John Libbey } \\
\text { Eurotext }\end{array}$ & 16 & Both & No & 0.33 \\
\hline 67. & Geriatric Care & PAGEPress & 4 & Online & Yes & \\
\hline
\end{tabular}


medRxiv preprint doi: https://doi.org/10.1101/19012278; this version posted November 22, 2019. The copyright holder for this preprint (which was not certified by peer review) is the author/funder, who has granted medRxiv a license to display the preprint in perpetuity.

It is made available under a CC-BY-ND 4.0 International license.

\begin{tabular}{|c|c|c|c|c|c|c|c|}
\hline & $2465-1109 ; 2465-1397$ & & & & & & \\
\hline 68. & $\begin{array}{l}\text { Geriatric Nursing } \\
0197-4572\end{array}$ & Elsevier & 38 & Both & $\begin{array}{l}\text { Optional; } \\
\text { APC } \$ 2000\end{array}$ & 1.142 & \\
\hline 69. & $\begin{array}{l}\frac{\text { Geriatric Orthopaedic }}{\text { Surgery \& Rehabilitation }} \\
2151-4593 ; 21514593\end{array}$ & $\begin{array}{l}\text { SAGE } \\
\text { Publications }\end{array}$ & 8 & Both & $\begin{array}{l}\text { Yes; APC } \\
\$ 1200\end{array}$ & 0.291 & \\
\hline 70. & $\frac{\text { Geriatrics }}{2308-3417}$ & MDPI & 2 & Online & $\begin{array}{l}\text { Yes; No APC } \\
\text { in } 2018\end{array}$ & & $\begin{array}{l}\text { Possibly } \\
\text { (Beall's List) }\end{array}$ \\
\hline 71. & $\frac{\frac{\text { Geriatrics and }}{\text { Gerontology }}}{\frac{\text { International }}{1447-0594}}$ & Wiley & 18 & Online & $\begin{array}{l}\text { Optional; } \\
\text { APC } \$ 3000\end{array}$ & 2.351 & \\
\hline 72. & $\frac{\text { Gerodontology }}{1741-2358}$ & Wiley & 35 & Both & $\begin{array}{l}\text { Optional; } \\
\text { APC } \$ 2500\end{array}$ & 1.681 & \\
\hline 73. & $\frac{\text { GeroInfo }}{1816-8450}$ & Medigraphic & 13 & Online & Yes & & \\
\hline 74. & $\frac{\text { Gerokomos }}{1134-928 \mathrm{X}}$ & $\begin{array}{l}\text { Ronda de Sant } \\
\text { Pere }\end{array}$ & 28 & Both & Yes & 0.4 & \\
\hline 75. & $\frac{\text { Gerontechnology }}{1569-1101}$ & \begin{tabular}{|l|} 
International \\
Society for \\
Gerontechnology
\end{tabular} & 17 & Both & $\begin{array}{l}\text { Yes; 1-year } \\
\text { embargo }\end{array}$ & 0.2 & \\
\hline 76. & $\begin{array}{l}\text { Gerontologist, The } \\
\text { 0016-9013; 1758-5341 }\end{array}$ & Oxford Academic & 57 & Both & No & 3.505 & \\
\hline 77. & $\frac{\text { Gérontologie et société }}{\text { 0151-0193; 2101-0218 }}$ & $\begin{array}{l}\text { Caisse nationale } \\
\text { d'assurance } \\
\text { vieillesse }\end{array}$ & 40 & Both & No & 0 & \\
\hline 78. & $\begin{array}{l}\text { Gerontology } \\
\text { 0304-324X; 1423-0003 }\end{array}$ & S. Karger AG & 63 & Both & $\begin{array}{l}\text { Optional; } \\
\text { APC } 3000 \\
(\mathrm{CHF})\end{array}$ & 4.252 & \\
\hline 79. & $\begin{array}{l}\frac{\text { Gerontology and }}{\text { Geriatric Medicine }} \\
\text { 2333-7214 }\end{array}$ & $\begin{array}{l}\text { SAGE } \\
\text { Publications }\end{array}$ & 3 & Online & Yes; $\$ 1200$ & & \\
\hline 80. & $\begin{array}{l}\frac{\text { Gerontology and }}{\text { Geriatrics }} \\
\text { 0014-424X }\end{array}$ & $\begin{array}{l}\text { Excerpta Medica } \\
\text { Foundation }\end{array}$ & 61 & Print & Unknown & & \\
\hline 81. & $\begin{array}{l}\frac{\text { Gerontology \& Geriatrics }}{\text { Education }} \\
0270-1960 ; 1545-3847\end{array}$ & $\begin{array}{l}\text { Taylor \& Francis } \\
\text { Online }\end{array}$ & 38 & Both & $\begin{array}{l}\text { Optional; } \\
\text { APC } \$ 2950\end{array}$ & 0.63 & \\
\hline 82. & $\begin{array}{l}\text { Gerontology \& } \\
\text { Geriatrics: Research }\end{array}$ & $\begin{array}{l}\text { Austin Publishing } \\
\text { Group }\end{array}$ & 2 & Online & $\begin{array}{l}\text { Yes; APC } \\
\$ 1800\end{array}$ & & $\begin{array}{l}\text { Yes (Beall's } \\
\text { List) }\end{array}$ \\
\hline 83. & $\begin{array}{l}\text { GeroPsych: The Journal } \\
\text { of Gerontopsychology } \\
\text { and Geriatric Psychiatry } \\
\text { 1662-9647; 1662-971X }\end{array}$ & Hogrefe AG & 30 & Both & $\begin{array}{l}\text { Optional; } \\
\text { APC } \$ 3000\end{array}$ & 0.36 & \\
\hline 84. & $\begin{array}{l}\text { GeroScience: Official } \\
\text { Journal of the American } \\
\text { Aging Association } \\
\text { (formerly AGE) } \\
2509-2715 ; 2509-2723\end{array}$ & Springer Nature & 39 & Both & $\begin{array}{l}\text { Optional; } \\
\text { APC } \$ 3000\end{array}$ & 2.123 & \\
\hline 85. & $\frac{\text { Global Ageing Journal }}{1729-3472}$ & $\begin{array}{l}\text { International } \\
\text { Federation on } \\
\text { Ageing }\end{array}$ & 9 & Online & Unknown & & \\
\hline
\end{tabular}


medRxiv preprint doi: https://doi.org/10.1101/19012278; this version posted November 22, 2019. The copyright holder for this preprint (which was not certified by peer review) is the author/funder, who has granted medRxiv a license to display the preprint in perpetuity.

It is made available under a CC-BY-ND 4.0 International license .

\begin{tabular}{|c|c|c|c|c|c|c|c|}
\hline 86. & $\begin{array}{l}\text { Global Journal of } \\
\text { Geriatrics Nursing } \\
\text { 2309-303X; 2375-4079 }\end{array}$ & $\begin{array}{l}\text { New Century } \\
\text { Science Press LLC }\end{array}$ & 5 & Online & $\begin{array}{l}\text { Yes; APC } \\
\$ 160\end{array}$ & & $\begin{array}{l}\text { Yes (Beall's } \\
\text { List) }\end{array}$ \\
\hline 87. & $\frac{\mathrm{GM}}{0268-201 X}$ & $\begin{array}{l}\text { Pavilion } \\
\text { Publishing and } \\
\text { Media }\end{array}$ & 47 & Both & Yes & & \\
\hline 88. & $\frac{\text { Healthy Aging Research }}{2261-7434}$ & Wolters Kluwer & 7 & Online & $\begin{array}{l}\text { Yes; APC } \\
\$ 1500\end{array}$ & & \\
\hline 89. & $\begin{array}{l}\text { Healthy Years (UCLA } \\
\text { Medical Center) }\end{array}$ & $\begin{array}{l}\text { Belvoir Media } \\
\text { Group LLC }\end{array}$ & 14 & Both & No & & \\
\hline 90. & $\begin{array}{l}\text { Immunity \& Ageing: I \& } \\
\text { A } \\
1742-4933\end{array}$ & IFP Media & 14 & Online & $\begin{array}{l}\text { Yes; APC } \\
\$ 2145\end{array}$ & 2.216 & \\
\hline 91. & $\begin{array}{l}\text { Indian Journal of } \\
\text { Geriatric Care } \\
\text { 0974-3405; 0974-2484 }\end{array}$ & $\begin{array}{l}\text { Geriatric Society } \\
\text { of India }\end{array}$ & 5 & Print & No & & \\
\hline 92. & $\begin{array}{l}\frac{\text { Indian Journal of }}{\text { Gerontology }} \\
\text { 0971-4189 }\end{array}$ & $\begin{array}{l}\text { Gerontology } \\
\text { India }\end{array}$ & 32 & Both & No & & \\
\hline 93. & $\frac{\text { Innovation in Aging }}{2399-5300}$ & Oxford Academic & 1 & Online & $\begin{array}{l}\text { Yes; APC } \\
\$ 2500\end{array}$ & & \\
\hline 94. & $\begin{array}{l}\text { Interdisciplinary Topics } \\
\frac{\text { in Gerontology and }}{\text { Geriatrics }} \\
2297-3508 ; 2297-3486\end{array}$ & Karger & 42 & Both & No & 2 & \\
\hline 95. & $\begin{array}{l}\text { International Journal of } \\
\text { Ageing and Later Life } \\
1652-8670\end{array}$ & $\begin{array}{l}\text { Linköping } \\
\text { University } \\
\text { Electronic Press }\end{array}$ & 11 & Online & Yes & 0.483 & \\
\hline 96. & $\begin{array}{l}\text { International Journal of } \\
\text { Aging and Human } \\
\text { Development, The } \\
\text { 0091-4150; 1541-3535 }\end{array}$ & $\begin{array}{l}\text { SAGE } \\
\text { Publications }\end{array}$ & 85 & Both & $\begin{array}{l}\text { Optional; } \\
\text { APC } \$ 3000\end{array}$ & 0.881 & \\
\hline 97. & $\begin{array}{l}\text { International Journal of } \\
\frac{\text { Aging and Society }}{2160-1909 ; 2160-1917}\end{array}$ & $\begin{array}{l}\text { Common Ground } \\
\text { Research } \\
\text { Networks }\end{array}$ & 7 & Both & $\begin{array}{l}\text { Optional; } \\
\text { APC } \$ 250\end{array}$ & & \\
\hline 98. & $\begin{array}{l}\text { International Journal of } \\
\text { Alzheimers \& } \\
\text { Neurological Disorders }\end{array}$ & BioAccent & 1 & Online & $\begin{array}{l}\text { Yes: APC } \\
\$ 750 / \$ 500\end{array}$ & & $\begin{array}{l}\text { Yes: (Beall's } \\
\text { List) }\end{array}$ \\
\hline 99. & $\begin{array}{l}\frac{\text { International Journal of }}{\text { Alzheimer's Disease }} \\
\text { 2090-8024; 2090-0252 }\end{array}$ & Hindawi Limited & 9 & Both & $\begin{array}{l}\text { Yes; APC } \\
\$ 750\end{array}$ & 0.787 & \\
\hline 100. & $\begin{array}{l}\text { International Journal of } \\
\text { Education and Ageing } \\
2044-5458\end{array}$ & $\begin{array}{l}\text { Association for } \\
\text { Education \& } \\
\text { Ageing (AEA) }\end{array}$ & 4 & Print & No & & \\
\hline 101. & $\begin{array}{l}\text { International Journal of } \\
\text { Geriatric Psychiatry } \\
\text { 1099-1166 }\end{array}$ & Wiley & 32 & Both & $\begin{array}{l}\text { Optional; } \\
\text { APC } \$ 3550\end{array}$ & 3.018 & \\
\hline 102. & $\begin{array}{l}\text { International Journal of } \\
\text { Gerontology } \\
1873-9598\end{array}$ & Elsevier & 11 & Online & $\begin{array}{l}\text { Yes; APC } \\
\$ 200-700\end{array}$ & 0.396 & \\
\hline 103. & International Journal of & Wiley & 13 & Online & Optional; & 1.333 & \\
\hline
\end{tabular}


medRxiv preprint doi: https://doi.org/10.1101/19012278; this version posted November 22, 2019. The copyright holder for this preprint (which was not certified by peer review) is the author/funder, who has granted medRxiv a license to display the preprint in perpetuity.

It is made available under a CC-BY-ND 4.0 International license.

\begin{tabular}{|c|c|c|c|c|c|c|c|}
\hline & $\begin{array}{l}\text { Older People Nursing } \\
\text { 1748-3735; 1748-3743 }\end{array}$ & & & & APC $\$ 2500$ & & \\
\hline 104. & $\frac{\frac{\text { International }}{\text { Psychogeriatrics }}}{1041-6102 ; 1741-203 X}$ & $\begin{array}{l}\text { Cambridge } \\
\text { University Press }\end{array}$ & 29 & Both & $\begin{array}{l}\text { Optional; } \\
\text { APC } \$ 2835\end{array}$ & 2.423 & \\
\hline 105. & $\begin{array}{l}\frac{\text { Internet Journal of }}{\text { Geriatrics and }} \\
\text { Gerontology, The } \\
1937-8211\end{array}$ & $\begin{array}{l}\text { Internet Scientific } \\
\text { Publications }\end{array}$ & 9 & Online & $\begin{array}{l}\text { Yes; } \\
\text { Publication } \\
\text { fee } \$ 275\end{array}$ & & $\begin{array}{l}\text { Yes (Beall's } \\
\text { List) }\end{array}$ \\
\hline 106. & $\begin{array}{l}\text { Japanese Journal of } \\
\text { Geriatrics } \\
\text { 0300-9173 }\end{array}$ & $\begin{array}{l}\text { The Japanese } \\
\text { Geriatrics Society }\end{array}$ & 55 & Online & Yes & 0.12 & \\
\hline 107. & $\begin{array}{l}\text { Journal of Aging and } \\
\text { Geriatric Medicine } \\
\text { 2576-3946 }\end{array}$ & SciTechnol & 1 & Online & $\begin{array}{l}\text { Yes; APC } \\
\$ 949\end{array}$ & & $\begin{array}{l}\text { Yes (Beall's } \\
\text { List) }\end{array}$ \\
\hline 108. & $\begin{array}{l}\text { Journal of Aging and } \\
\text { Geriatric Psychiatry }\end{array}$ & Allied Academics & 1 & Online & $\begin{array}{l}\text { Yes; APC } \\
\$ 719\end{array}$ & & \\
\hline 109. & $\begin{array}{l}\text { Journal of Aging and } \\
\text { Health } \\
\text { 0898-2643; 1552-6887 }\end{array}$ & $\begin{array}{l}\text { SAGE } \\
\text { Publications }\end{array}$ & 29 & Both & $\begin{array}{l}\text { Optional; } \\
\text { APC } \$ 3000\end{array}$ & 2.168 & \\
\hline 110. & $\begin{array}{l}\text { Journal of Aging Life } \\
\text { Care }\end{array}$ & $\begin{array}{l}\text { Aging Life Care } \\
\text { Association }\end{array}$ & 12 & Online & Unknown & & \\
\hline 111. & $\begin{array}{l}\overline{\text { Journal of Aging and }} \\
\frac{\text { Long-Term Care }}{2619-9017 ; 2618-6535}\end{array}$ & $\begin{array}{l}\text { National } \\
\text { Association of } \\
\text { Social and } \\
\text { Applied } \\
\text { Gerontology }\end{array}$ & 1 & Both & No & & \\
\hline 112. & $\begin{array}{l}\frac{\text { Journal of Aging and }}{\text { Physical Activity }} \\
\text { 1063-8652; 1543-267X }\end{array}$ & Atypon Literatum & 25 & Both & $\begin{array}{l}\text { Optional; } \\
\text { APC } \$ 2000\end{array}$ & 1.937 & \\
\hline 113. & $\begin{array}{l}\frac{\text { Journal of Aging }}{2090-2204} \\
\frac{\text { Research }}{2090}\end{array}$ & Hindawi Limited & 8 & Both & $\begin{array}{l}\text { Yes; APC } \\
\$ 750\end{array}$ & 0.769 & \\
\hline 114. & $\begin{array}{l}\text { Journal of Aging } \\
\text { Research \& Clinical } \\
\frac{\text { Practice }}{2273-421 X}\end{array}$ & IAGG \& GARN & 6 & Online & $\begin{array}{l}\text { Yes; APC } \\
\text { Unknown }\end{array}$ & & \\
\hline 115. & $\begin{array}{l}\text { Journal of Aging } \\
\text { Research and Healthcare } \\
2474-7785\end{array}$ & Open Access Pub & 2 & Online & $\begin{array}{l}\text { Yes, APC } \\
\$ 1,200\end{array}$ & & $\begin{array}{l}\text { Yes (Beall's } \\
\text { List) }\end{array}$ \\
\hline 116. & $\frac{\text { Iournal of Aging Science }}{2329-8847}$ & $\begin{array}{l}\text { OMICS } \\
\text { International }\end{array}$ & 5 & Online & $\begin{array}{l}\text { Yes; APC } \\
\$ 1000\end{array}$ & 0.50 & $\begin{array}{l}\text { Yes (Beall's } \\
\text { List) }\end{array}$ \\
\hline 117. & $\begin{array}{l}\text { Journal of Aging and } \\
\text { Social Policy } \\
\text { 0895-9420; } 1545-0821\end{array}$ & $\begin{array}{l}\text { Taylor \& Francis } \\
\text { Online }\end{array}$ & 29 & Both & $\begin{array}{l}\text { Optional; } \\
\text { APC } \$ 2950\end{array}$ & 1.256 & \\
\hline 118. & $\begin{array}{l}\text { Journal of Aging Studies } \\
0890-4065\end{array}$ & Elsevier & 44 & Both & $\begin{array}{l}\text { Optional; } \\
\text { APC } \$ 1800\end{array}$ & 1.248 & \\
\hline 119. & $\begin{array}{l}\text { Journal of Alzheimer's } \\
\frac{\text { Disease }}{1387-2877 ; 1875-8908}\end{array}$ & IOS Press & 64 & Both & $\begin{array}{l}\text { Optional; } \\
\text { APC } \$ 1450 \\
\text { (plus } \$ 700 \\
\text { charge) }\end{array}$ & 3.731 & \\
\hline 120. & Journal of Alzheimer's & OMICS & 7 & Online & Yes; APC & 3.03 & Yes (Beall's \\
\hline
\end{tabular}


medRxiv preprint doi: https://doi.org/10.1101/19012278; this version posted November 22, 2019. The copyright holder for this preprint (which was not certified by peer review) is the author/funder, who has granted medRxiv a license to display the preprint in perpetuity.

It is made available under a CC-BY-ND 4.0 International license .

\begin{tabular}{|c|c|c|c|c|c|c|c|}
\hline & $\begin{array}{l}\frac{\text { Disease and }}{\text { Parkinsonism }} \\
2161-0460\end{array}$ & International & & & $\$ 3162$ & & List) \\
\hline 121. & $\begin{array}{l}\text { Journal of Alzheimer's } \\
\text { Disease Reports } \\
2542-4823\end{array}$ & IOS Press & 2 & Online & $\begin{array}{l}\text { Yes; APC } \\
\$ 725 \text { for first } \\
\text { two years of } \\
\text { publication }\end{array}$ & & \\
\hline 122. & $\begin{array}{l}\text { Journal of Applied } \\
\text { Gerontology } \\
0733-4648 ; 1552-4523\end{array}$ & $\begin{array}{l}\text { SAGE } \\
\text { Publications }\end{array}$ & 36 & Both & $\begin{array}{l}\text { Optional; } \\
\text { APC } \$ 3000\end{array}$ & 1.638 & \\
\hline 123. & $\begin{array}{l}\frac{\text { Journal of Clinical }}{\text { Gerontology and }} \\
\frac{\text { Geriatrics }}{2210-8335}\end{array}$ & $\begin{array}{l}\text { Content Ed Net } \\
\text { Taiwan Limited } \\
\text { (formerly } \\
\text { Elsevier) }\end{array}$ & 8 & Online & Yes & 0.82 & \\
\hline 124. & $\begin{array}{l}\text { Iournal of Cross-Cultural } \\
\text { Gerontology } \\
\text { 0169-3816; 1573-0719 }\end{array}$ & Springer Nature & 32 & Both & $\begin{array}{l}\text { Optional; } \\
\text { APC } \$ 3000\end{array}$ & 0.734 & \\
\hline 125. & $\frac{\text { Iournal of Dementia Care }}{1351-8372}$ & $\begin{array}{l}\text { Hawker } \\
\text { Publications Ltd. }\end{array}$ & 25 & Both & No & 0.05 & \\
\hline 126. & $\begin{array}{l}\text { Journal of Elder Abuse \& } \\
\text { Neglect } \\
0894-6566 ; 1540-4129\end{array}$ & $\begin{array}{l}\text { Taylor \& Francis } \\
\text { Online }\end{array}$ & 29 & Both & $\begin{array}{l}\text { Optional; } \\
\text { APC } \$ 2950\end{array}$ & 1.36 & \\
\hline 127. & $\begin{array}{l}\text { Journal of Frailty \& } \\
\frac{\text { Aging, The }}{2260-1341 ; 2273-4309}\end{array}$ & $\begin{array}{l}\text { Toulouse : Serdi } \\
\text { Edition }\end{array}$ & 7 & Both & $\begin{array}{l}\text { Some Free } \\
\text { Access } \\
\text { Available; } \\
\text { PF } \$ 328 / \text { pg } \\
\text { after } 2 \text { pages }\end{array}$ & 0.573 & \\
\hline 128. & $\frac{\text { Journal of Geriatric }}{\frac{\text { Cardiology }}{1671-5411}}$ & Science Press & 14 & Online & $\begin{array}{l}\text { Yes; APC } \\
\$ 1650\end{array}$ & 1.806 & \\
\hline 129. & $\frac{\text { Journal of Geriatric }}{\text { Mental Health }}$ & $\begin{array}{l}\text { Indian } \\
\text { Association for } \\
\text { Geriatric Mental } \\
\text { Health }\end{array}$ & 4 & Both & Yes & & \\
\hline 130. & $\frac{\text { Journal of Geriatric }}{\text { Oncology }}$ & Elsevier & 9 & Both & $\begin{array}{l}\text { Optional; } \\
\text { APC } \$ 1700\end{array}$ & 2.852 & \\
\hline 131. & $\frac{\text { Iournal of Geriatric }}{\text { Physical Therapy }}$ & Wolters Kluwer & 40 & Both & $\begin{array}{l}\text { Optional; } \\
\text { APC } \$ 2700\end{array}$ & 1.833 & \\
\hline 132. & $\frac{\frac{\text { Journal of Geriatric }}{\text { Psychiatry and }}}{\frac{\text { Neurology }}{0891-9887 ; 1552-5708}}$ & $\begin{array}{l}\text { SAGE } \\
\text { Publications }\end{array}$ & 30 & Both & No & 2.109 & \\
\hline 133. & $\begin{array}{l}\text { Journal of Gerontological } \\
\text { Nursing } \\
0098-9134 ; 1938-243 X\end{array}$ & Healio & 43 & Both & $\begin{array}{l}\text { Optional; } \\
\text { APC } \$ 1500\end{array}$ & 0.827 & \\
\hline 134. & $\begin{array}{l}\text { Journal of Gerontological } \\
\text { Social Work } \\
0163-4372 ; 1540-4048\end{array}$ & $\begin{array}{l}\text { Taylor \& Francis } \\
\text { Online }\end{array}$ & 60 & Both & $\begin{array}{l}\text { Optional; } \\
\text { APC } \$ 2950\end{array}$ & 0.513 & \\
\hline 135. & $\begin{array}{l}\text { Journal of Gerontology } \\
\text { and Geriatrics (formerly }\end{array}$ & $\begin{array}{l}\text { Pacini Editore } \\
\text { SRL }\end{array}$ & 66 & Online & Yes & 0.121 & \\
\hline
\end{tabular}


medRxiv preprint doi: https://doi.org/10.1101/19012278; this version posted November 22, 2019. The copyright holder for this preprint (which was not certified by peer review) is the author/funder, who has granted medRxiv a license to display the preprint in perpetuity.

It is made available under a CC-BY-ND 4.0 International license .

\begin{tabular}{|c|c|c|c|c|c|c|c|}
\hline & $\begin{array}{l}\text { Giornale di Gerontologia) } \\
2499-6564\end{array}$ & & & & & & \\
\hline 136. & $\begin{array}{l}\frac{\text { Lournal of Gerontology }}{\text { and Geriatric Research }} \\
\frac{2167-7182}{}\end{array}$ & $\begin{array}{l}\text { OMICS } \\
\text { International }\end{array}$ & 6 & Online & $\begin{array}{l}\text { Yes; APC } \\
\$ 2719\end{array}$ & 0.92 & $\begin{array}{l}\text { Yes (Beall's } \\
\text { List) }\end{array}$ \\
\hline 137. & $\begin{array}{l}\text { Journal of Health and } \\
\text { Social Behavior } \\
0022-1465 ; 2150-6000\end{array}$ & $\begin{array}{l}\text { SAGE } \\
\text { Publications }\end{array}$ & 59 & Both & No & 1.949 & \\
\hline 138. & $\begin{array}{l}\text { Journal of Housing for } \\
\text { the Elderly } \\
\underline{0276-3893 ; 1540-353 X}\end{array}$ & $\begin{array}{l}\text { Taylor \& Francis } \\
\text { Online }\end{array}$ & 32 & Both & $\begin{array}{l}\text { Optional; } \\
\text { APC } \$ 2950\end{array}$ & 0.186 & \\
\hline 139. & $\begin{array}{l}\frac{\text { Lournal of }}{\text { Intergenerational }} \\
\frac{\text { Relationships }}{1535-0770 ; 1535-0932}\end{array}$ & $\begin{array}{l}\text { Taylor \& Francis } \\
\text { Online }\end{array}$ & 16 & Both & $\begin{array}{l}\text { Optional; } \\
\text { APC } \$ 2950\end{array}$ & 0.44 & \\
\hline 140 & $\begin{array}{l}\text { Journal of Korean } \\
\frac{\text { Geriatric Psychiatry }}{1226-6329}\end{array}$ & $\begin{array}{l}\text { Sŏul : Taehan } \\
\text { Noin Chŏngsin } \\
\text { Ǔihakhoe }\end{array}$ & 22 & Print & No & & \\
\hline 141. & $\begin{array}{l}\text { Journal of Mental Health } \\
\text { and Aging }\end{array}$ & Allied Academies & 2 & Online & $\begin{array}{l}\text { Yes; } \\
\text { APC } \$ 519\end{array}$ & & $\begin{array}{l}\text { Yes, (Beall's } \\
\text { List) }\end{array}$ \\
\hline 142. & $\begin{array}{l}\text { Journal of Mid-Life } \\
\frac{\text { Health }}{\text { 0976-7800; 0976-7819 }}\end{array}$ & $\begin{array}{l}\text { Medknow } \\
\text { Publications }\end{array}$ & 9 & Both & Yes & 0.308 & \\
\hline 143. & $\begin{array}{l}\text { Journal of Nutrition, } \\
\text { Health \& Aging, The } \\
\text { 1279-7707; 1760-4788 }\end{array}$ & Springer Nature & 21 & Both & $\begin{array}{l}\text { Optional; } \\
\text { APC } \$ 3000\end{array}$ & 2.868 & \\
\hline 144 & $\begin{array}{l}\frac{\text { Journal of Nutrition in }}{\text { Gerontology and }} \\
\frac{\text { Geriatrics }}{2155-1197 ; 2155-1200}\end{array}$ & $\begin{array}{l}\text { Taylor \& Francis } \\
\text { Online }\end{array}$ & 36 & Both & $\begin{array}{l}\text { Optional; } \\
\text { APC } \$ 2950\end{array}$ & 1.16 & \\
\hline 145. & $\begin{array}{l}\text { Journal of Parkinsonism } \\
\text { and Restless Legs } \\
\frac{\text { Syndrome }}{1927-7733}\end{array}$ & Dovepress & 8 & Online & APC $\$ 1,780$ & & \\
\hline 146. & $\frac{\text { Journal of Parkinson's }}{\text { Disease }}$ & IOS Press & 7 & Online & $\begin{array}{l}\text { Optional; } \\
\text { APC } \$ 1450\end{array}$ & 1.151 & $\begin{array}{l}\text { Yes (Beall's } \\
\text { List) }\end{array}$ \\
\hline 147. & $\begin{array}{l}\text { Journal of Population } \\
\text { Ageing } \\
\text { 1874-7884; 1874-7876 }\end{array}$ & Springer Nature & 10 & Both & $\begin{array}{l}\text { Optional; } \\
\text { APC } \$ 3000\end{array}$ & & \\
\hline 148. & $\begin{array}{l}\text { Journal of Religion, } \\
\text { Spirituality \& Aging } \\
\text { (formerly Journal of } \\
\text { Religious Gerontology) } \\
\text { 1552-8030; } 1552-8049\end{array}$ & $\begin{array}{l}\text { Taylor \& Francis } \\
\text { Online }\end{array}$ & 29 & Both & $\begin{array}{l}\text { Optional; } \\
\text { APC } \$ 2950\end{array}$ & 0.179 & \\
\hline 149. & $\begin{array}{l}\text { Journal of Social Work in } \\
\text { End-of-Life \& Palliative } \\
\text { Care } \\
1552-4256 ; 1552-4264\end{array}$ & $\begin{array}{l}\text { Taylor \& Francis } \\
\text { Online }\end{array}$ & 13 & Both & $\begin{array}{l}\text { Optional; } \\
\text { APC } \$ 2950\end{array}$ & 0.345 & \\
\hline 150. & $\begin{array}{l}\text { Journal of Women \& } \\
\text { Aging } \\
0895-2841 ; 1540-7322\end{array}$ & $\begin{array}{l}\text { Taylor \& Francis } \\
\text { Online }\end{array}$ & 29 & Both & $\begin{array}{l}\text { Optional; } \\
\text { APC } \$ 2950\end{array}$ & 0.74 & \\
\hline
\end{tabular}


medRxiv preprint doi: https://doi.org/10.1101/19012278; this version posted November 22, 2019. The copyright holder for this preprint (which was not certified by peer review) is the author/funder, who has granted medRxiv a license to display the preprint in perpetuity.

It is made available under a CC-BY-ND 4.0 International license .

\begin{tabular}{|c|c|c|c|c|c|c|c|}
\hline 151. & $\begin{array}{l}\text { Journal of the American } \\
\text { Geriatrics Society } \\
\text { 0002-8614; } 1532-5415\end{array}$ & Wiley & 66 & Both & $\begin{array}{l}\text { Optional; } \\
\text { APC } \$ 4300\end{array}$ & 4.388 & \\
\hline 152. & $\begin{array}{l}\text { Journal of the American } \\
\text { Medical Directors } \\
\text { Association (JAMDA), } \\
\text { The; The Journal of Post- } \\
\text { Acute and Long-Term } \\
\text { Care Medicine } \\
\text { 1525-8610; } 1538-9375\end{array}$ & Elsevier & 19 & Both & $\begin{array}{l}\text { Optional; } \\
\text { APC } \$ 3600\end{array}$ & 5.325 & \\
\hline 153. & $\begin{array}{l}\text { Journal of the Economics } \\
\text { of Ageing } \\
2212-828 X\end{array}$ & Elsevier & 10 & Both & $\begin{array}{l}\text { Optional; } \\
\text { APC } \$ 1100\end{array}$ & 0.631 & \\
\hline 154. & $\begin{array}{l}\text { Journal of the Indian } \\
\text { Academy of Geriatrics } \\
\text { 0974-2484 }\end{array}$ & $\begin{array}{l}\text { Indian Academy } \\
\text { of Geriatrics }\end{array}$ & 13 & Online & Yes & 0.111 & \\
\hline 155. & $\begin{array}{l}\text { Journals of Gerontology, } \\
\text { The. Series A, Biological } \\
\text { Sciences and Medical } \\
\text { Sciences } \\
\text { 1079-5006; 1758-535X }\end{array}$ & Oxford Academic & 72 & Both & No & 4.902 & \\
\hline 156. & $\begin{array}{l}\text { Journals of Gerontology, } \\
\text { The. Series B } \\
\text { Psychological Sciences } \\
\begin{array}{l}\text { and Social Sciences } \\
\text { 1079-5014; } 1758-5368\end{array}\end{array}$ & Oxford Academic & 72 & Both & No & 3.064 & \\
\hline 157. & $\frac{\text { Maturitas }}{0378-5122}$ & Elsevier & 107 & Both & $\begin{array}{l}\text { Optional; } \\
\text { APC } \$ 3200\end{array}$ & 3.255 & \\
\hline 158. & $\begin{array}{l}\text { Mechanisms of Ageing } \\
\text { and Development } \\
\text { 0047-6374 }\end{array}$ & Elsevier & 173 & Both & $\begin{array}{l}\text { Optional; } \\
\text { APC } \$ 2650\end{array}$ & 3.087 & \\
\hline 159. & $\begin{array}{l}\frac{\text { MedCrave Online }}{\text { Journal (MOI) of }} \\
\frac{\text { Gerontology \& Geriatrics }}{2574-8130}\end{array}$ & MedCrave Group & 2 & Online & $\begin{array}{l}\text { Yes; APC } \\
\$ 219-989 \\
\text { (dependent } \\
\text { upon income } \\
\text { and } \\
\text { submission) }\end{array}$ & & $\begin{array}{l}\text { Yes (Beall's } \\
\text { List) }\end{array}$ \\
\hline 160. & $\begin{array}{l}\frac{\text { Middle East Journal of }}{\text { Age and Ageing }} \\
1449-8677\end{array}$ & $\begin{array}{l}\text { medi+WORLD } \\
\text { International }\end{array}$ & 14 & Online & $\begin{array}{l}\text { Yes; APC } \\
\$ 350\end{array}$ & & \\
\hline 161. & $\frac{\frac{\text { Molecular }}{\text { Neurodegeneration }}}{1750-1326}$ & Springer Nature & 13 & Online & $\begin{array}{l}\text { Yes; APC } \\
\$ 2580\end{array}$ & 3.418 & \\
\hline 162. & $\begin{array}{l}\text { NBER Bulletin on Aging } \\
\text { and Health, the }\end{array}$ & $\begin{array}{l}\text { The National } \\
\text { Bureau of } \\
\text { Economic } \\
\text { Research }\end{array}$ & 31 & Online & Yes & & \\
\hline 163. & $\begin{array}{l}\text { Neurobiology of Aging } \\
0197-4580\end{array}$ & Elsevier & 60 & Both & $\begin{array}{l}\text { Optional; } \\
\text { APC } \$ 3300\end{array}$ & 5.117 & \\
\hline 164. & $\begin{array}{l}\frac{\text { Neurodegenerative }}{\text { Diseases }} \\
1660-2854 ; 1660-2862\end{array}$ & Karger & 18 & Both & $\begin{array}{l}\text { Optional; } \\
\text { APC CHF } \\
3000\end{array}$ & 2.842 & \\
\hline
\end{tabular}


medRxiv preprint doi: https://doi.org/10.1101/19012278; this version posted November 22, 2019. The copyright holder for this preprint (which was not certified by peer review) is the author/funder, who has granted medRxiv a license to display the preprint in perpetuity.

It is made available under a CC-BY-ND 4.0 International license .

\begin{tabular}{|c|c|c|c|c|c|c|c|}
\hline 165. & $\begin{array}{l}\frac{\text { NPG Neurologie - }}{\text { Psychiatrie - Gériatrie }} \\
\frac{1627-4830}{}\end{array}$ & Elsevier BV & 18 & Both & $\begin{array}{l}\text { Optional; } \\
\text { EUR } 2000\end{array}$ & 0.19 & \\
\hline 166. & $\begin{array}{l}\frac{\text { NPJ Aging and }}{\text { Mechanisms of Disease }} \\
2056-3973\end{array}$ & Springer Nature & 52 articles & Online & $\begin{array}{l}\text { Yes; APC } \\
\$ 2800\end{array}$ & & \\
\hline 167. & $\begin{array}{l}\text { Nursing Older People } \\
\text { (formerly Elderly Care) } \\
\text { 1472-0795; 2047-8941 }\end{array}$ & $\mathrm{RCNi}$ & 29 & Both & $\begin{array}{l}\text { Optional; } \\
\text { APC } £ 1000\end{array}$ & & \\
\hline 168. & $\begin{array}{l}\text { Nutrition and Healthy } \\
\text { Aging (formerly } \\
\text { Nutrition and Aging) } \\
\text { 2451-9480; 2451-9502 }\end{array}$ & IOS Press & 4 & Print & $\begin{array}{l}\text { Yes; APC } \\
\$ 1250\end{array}$ & & $\begin{array}{l}\text { Yes (Beall's } \\
\text { List) }\end{array}$ \\
\hline 169. & $\begin{array}{l}\text { OMEGA - Journal of } \\
\text { Death and Dying } \\
0030-2228 ; 1541-3764\end{array}$ & $\begin{array}{l}\text { SAGE } \\
\text { Publications }\end{array}$ & 76 & Both & $\begin{array}{l}\text { Optional; } \\
\text { APC } \$ 3000\end{array}$ & 0.676 & \\
\hline 170. & $\begin{array}{l}\frac{\text { Oxidative Medicine \& }}{\text { Cellular Longevity }} \\
1942-0900\end{array}$ & Hindawi Limited & 10 & Both & $\begin{array}{l}\text { Yes; APC } \\
\$ 2000\end{array}$ & 4.593 & \\
\hline 171. & $\frac{\frac{\text { Parkinsonism and }}{\text { Related Disorders }}}{1353-8020}$ & Elsevier & 49 & Both & $\begin{array}{l}\text { Optional; } \\
\text { APC } \$ 3200\end{array}$ & 4.484 & \\
\hline 172. & $\frac{\text { Parkinson's Disease }}{2090-8083}$ & Hindawi Limited & 8 & Both & $\begin{array}{l}\text { Yes; APC } \\
\$ 1500\end{array}$ & 1.702 & \\
\hline 173. & $\begin{array}{l}\text { Pathobiology of Aging \& } \\
\text { Age-related Diseases } \\
2001-0001\end{array}$ & $\begin{array}{l}\text { Taylor \& Francis } \\
\text { Online }\end{array}$ & 7 & Online & Yes; No APC & 0.379 & \\
\hline 174. & $\begin{array}{l}\text { Physical \& Occupational } \\
\text { Therapy in Geriatrics } \\
\text { 0270-3181; 1541-3152 }\end{array}$ & $\begin{array}{l}\text { Taylor \& Francis } \\
\text { Online }\end{array}$ & 35 & Both & $\begin{array}{l}\text { Optional; } \\
\text { APC } \$ 2950\end{array}$ & 0.26 & \\
\hline 175. & $\begin{array}{l}\text { Post Reproductive } \\
\text { Health (formerly } \\
\text { Menopause } \\
\text { International) } \\
\text { 2053-3691; 2053-3705 }\end{array}$ & $\begin{array}{l}\text { SAGE } \\
\text { Publications }\end{array}$ & 23 & Both & No & 0.287 & \\
\hline 176. & $\begin{array}{l}\text { Productive Aging Special } \\
\text { Interest Section (formerly } \\
\text { Gerontology Special } \\
\text { Interest Section) }\end{array}$ & $\begin{array}{l}\text { The American } \\
\text { Occupational } \\
\text { Therapy } \\
\text { Association, Inc. }\end{array}$ & 40 (?) & Both & No & & \\
\hline 177. & $\begin{array}{l}\text { Psychogeriatrics } \\
\text { 1346-3500; } 1479-8301\end{array}$ & Wiley & 17 & Both & $\begin{array}{l}\text { Optional; } \\
\text { APC } \$ 2700\end{array}$ & 1.693 & \\
\hline 178. & $\begin{array}{l}\text { Psychology and Aging } \\
0882-7974 ; 1939-1498\end{array}$ & $\begin{array}{l}\text { American } \\
\text { Psychological } \\
\text { Association }\end{array}$ & 32 & Both & No & 2.812 & \\
\hline 179. & $\begin{array}{l}\text { Public Policy \& Aging } \\
\text { Report } \\
\text { 1055-3037; 2053-4892 }\end{array}$ & Oxford Academic & 27 & Both & No & & \\
\hline 180. & $\frac{\text { Quality in Ageing and }}{\text { Older Adults }}$ & $\begin{array}{l}\text { Emerald } \\
\text { Publishing } \\
\text { Limited }\end{array}$ & 18 & Both & $\begin{array}{l}\text { Optional; } \\
\text { APC } \$ 2000\end{array}$ & & \\
\hline 181. & $\frac{\text { Rejuvenation Research }}{1549-1684 ; 1557-8577}$ & $\begin{array}{l}\text { Mary Ann } \\
\text { Liebert, Inc. }\end{array}$ & 21 & Online & Optional & 2.827 & \\
\hline
\end{tabular}


medRxiv preprint doi: https://doi.org/10.1101/19012278; this version posted November 22, 2019. The copyright holder for this preprint (which was not certified by peer review) is the author/funder, who has granted medRxiv a license to display the preprint in perpetuity.

It is made available under a CC-BY-ND 4.0 International license .

\begin{tabular}{|c|c|c|c|c|c|c|c|}
\hline 182. & $\begin{array}{l}\text { Research on Aging } \\
0164-0275 ; 1552-7573\end{array}$ & $\begin{array}{l}\text { SAGE } \\
\text { Publications }\end{array}$ & 39 & Both & No & 1.214 & \\
\hline 183. & $\begin{array}{l}\frac{\text { Research in }}{\text { Gerontological Nursing }} \\
\text { 1940-4921; 1938-2464 }\end{array}$ & Healio & 10 & Both & $\begin{array}{l}\text { Optional; } \\
\text { APC } \$ 1500\end{array}$ & 0.717 & \\
\hline 184. & $\begin{array}{l}\frac{\text { Revista Brasileira de }}{\text { Ciências do }} \\
\text { Envelhecimento Humano } \\
1679-7930 ; 2317-6695\end{array}$ & UPF Editora & 14 & Online & Unknown & & \\
\hline 185. & $\begin{array}{l}\frac{\text { Revista Brasileira de }}{\text { Geriatria e Gerontologia }} \\
\text { 1981-2256 }\end{array}$ & $\begin{array}{l}\text { Open University } \\
\text { of the Third Age }\end{array}$ & 21 & Online & Yes & & \\
\hline 186. & $\begin{array}{l}\frac{\text { Revista Española de }}{\text { Geriatría y Gerontología }} \\
\text { 0211-139X }\end{array}$ & Elsevier España & 53 & Both & $\begin{array}{l}\text { Optional; 1- } \\
\text { year } \\
\text { embargo }\end{array}$ & 1.12 & \\
\hline 187. & $\begin{array}{l}\frac{\text { Revista Ibero-America de }}{\text { Saúde e Envelhecimento }} \\
2183-6663\end{array}$ & $\begin{array}{l}\text { The University of } \\
\text { Évora }\end{array}$ & 4 & Online & Yes & & \\
\hline 188. & $\begin{array}{l}\text { Seniors Housing \& Care } \\
\text { Journal } \\
1941-7187\end{array}$ & $\begin{array}{l}\text { National } \\
\text { Investment } \\
\text { Center for the } \\
\text { Seniors Housing } \\
\text { \& Care Industry }\end{array}$ & 25 & Print & Yes & & \\
\hline 189. & $\begin{array}{l}\text { SM Gerontology and } \\
\frac{\text { Geriatric Research }}{2576-5434}\end{array}$ & SM Online & 2 & Online & $\begin{array}{l}\text { Yes: APC } \\
\text { not given }\end{array}$ & & $\begin{array}{l}\text { Yes: (Beall's } \\
\text { List) }\end{array}$ \\
\hline 190. & $\begin{array}{l}\text { Tijdschrift voor } \\
\text { Gerontologie en Geriatrie } \\
0167-9228 ; 1875-6832\end{array}$ & Springer Nature & 49 & Both & No & 0.25 & \\
\hline 191. & $\begin{array}{l}\text { Tijdschrift voor } \\
\text { Ouderengeneeskunde }\end{array}$ & Verenso & 8 & Online & No & 0 & \\
\hline 192. & $\begin{array}{l}\frac{\text { Today's Geriatric }}{\text { Medicine }} \\
\text { 2328-0778; 2328-0786 }\end{array}$ & $\begin{array}{l}\text { Great Valley } \\
\text { Publishing }\end{array}$ & 10 & Both & Yes & & \\
\hline 193. & $\begin{array}{l}\frac{\text { Topics in Geriatric }}{\text { Rehabilitation }} \\
\text { 0882-7524; } 1550-2414\end{array}$ & $\begin{array}{l}\text { Lippincott, } \\
\text { Williams \& } \\
\text { Wilkins }\end{array}$ & 33 & Online & $\begin{array}{l}\text { Optional; } \\
\text { APC } \$ 4000\end{array}$ & 0.378 & \\
\hline 194. & $\begin{array}{l}\text { Translational Medicine of } \\
\text { Aging } \\
2468-5011\end{array}$ & $\begin{array}{l}\text { KeAi } \\
\text { Communications } \\
\text { Co. }\end{array}$ & 2 & Online & $\begin{array}{l}\text { Yes; APC } \\
\$ 980\end{array}$ & & \\
\hline 195. & $\begin{array}{l}\text { Turkish Journal of } \\
\text { Geriatrics (Turk Geriatri } \\
\frac{\text { Dergisi) }}{1304-2947 ; 1307-9948}\end{array}$ & $\begin{array}{l}\text { Turkish Geriatrics } \\
\text { Society }\end{array}$ & 21 & Online & Unknown & 0.17 & \\
\hline 196. & $\begin{array}{l}\text { Work, Aging and } \\
\text { Retirement } \\
\text { 2054-4642; 2054-4650 }\end{array}$ & Oxford Academic & 4 & Both & No & & \\
\hline 197. & $\begin{array}{l}\text { Working with Older } \\
\frac{\text { People }}{1366-3666}\end{array}$ & $\begin{array}{l}\text { Emerald } \\
\text { Publishing } \\
\text { Limited }\end{array}$ & 21 & Both & $\begin{array}{l}\text { Optional; } \\
\text { APC } \$ 2000\end{array}$ & 0.162 & \\
\hline
\end{tabular}


medRxiv preprint doi: https://doi.org/10.1101/19012278; this version posted November 22, 2019. The copyright holder for this preprint (which was not certified by peer review) is the author/funder, who has granted medRxiv a license to display the preprint in perpetuity.

It is made available under a CC-BY-ND 4.0 International license .

\begin{tabular}{|l|l|l|l|l|l|}
\hline Zeitschrift für & Springer Nature & 51 & Both & $\begin{array}{l}\text { Optional; } \\
\text { APC } \$ 3000\end{array}$ & 0.885 \\
\hline $\begin{array}{l}\text { Gerontologie und } \\
\text { Geriatrie }\end{array}$ & & & & & \\
\hline $\begin{array}{l}0948-6704 ; \\
1435-1269\end{array}$ & & & & \\
\hline
\end{tabular}

*The impact factor of the journal was listed when available on the journal website. The Scimago Journal Ranking was used when the impact factor was not available from the journal website. The impact factor should not be used as the only indicator of journal quality. 
medRxiv preprint doi: https://doi.org/10.1101/19012278; this version posted November 22, 2019. The copyright holder for this preprint (which was not certified by peer review) is the author/funder, who has granted medRxiv a license to display the preprint in perpetuity.

It is made available under a CC-BY-ND 4.0 International license.

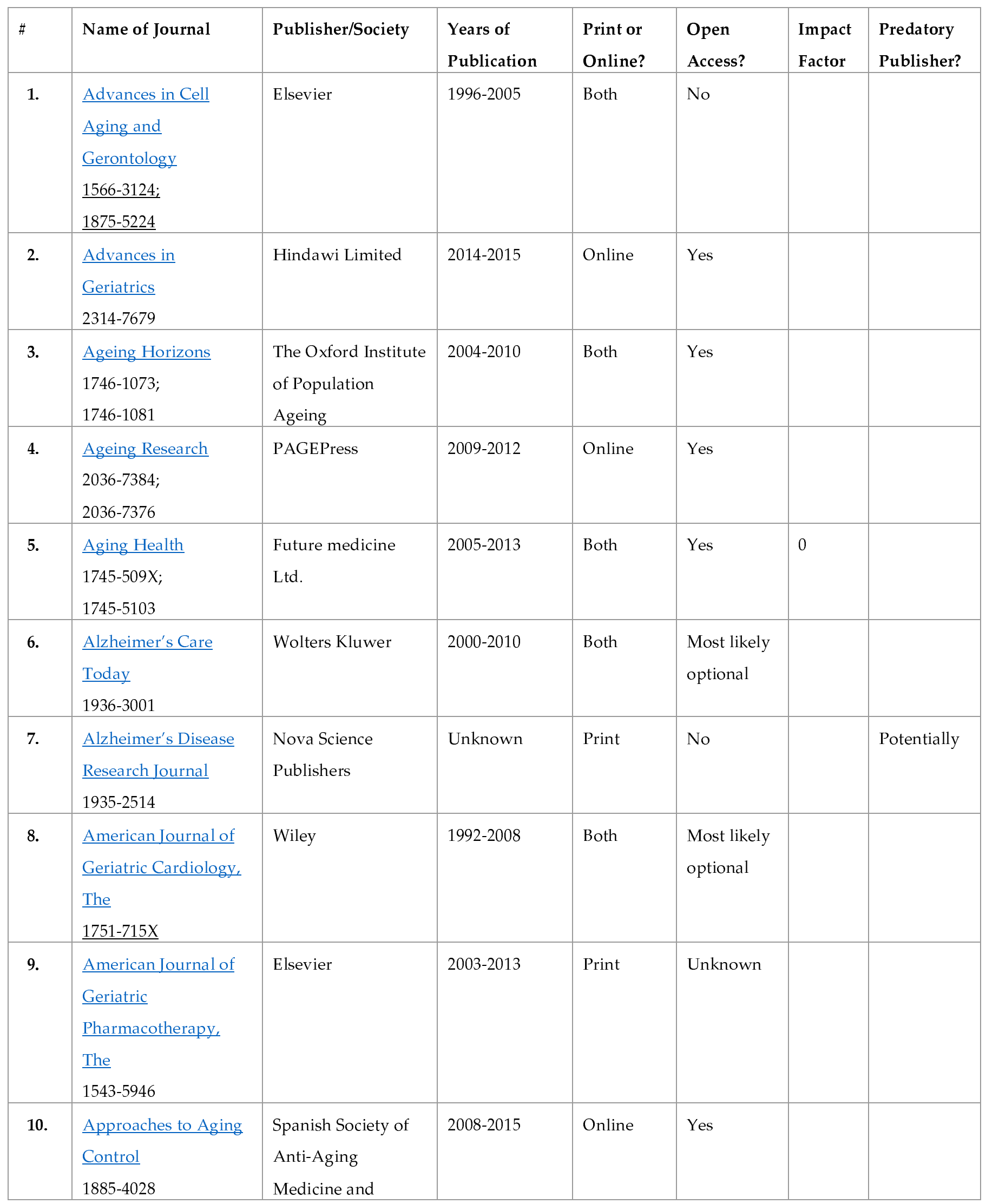


medRxiv preprint doi: https://doi.org/10.1101/19012278; this version posted November 22, 2019. The copyright holder for this preprint (which was not certified by peer review) is the author/funder, who has granted medRxiv a license to display the preprint in perpetuity.

It is made available under a CC-BY-ND 4.0 International license .

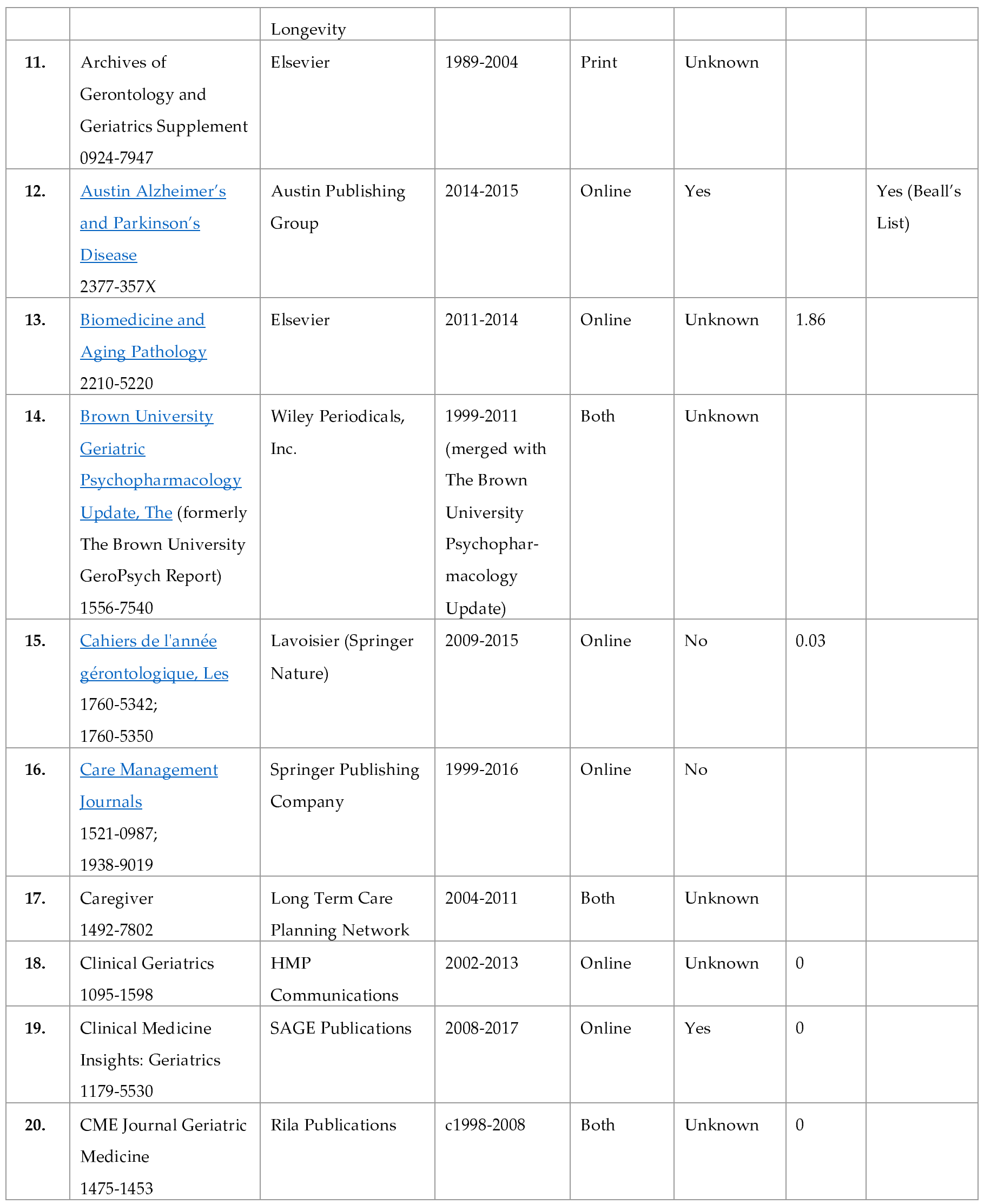


medRxiv preprint doi: https://doi.org/10.1101/19012278; this version posted November 22, 2019. The copyright holder for this preprint (which was not certified by peer review) is the author/funder, who has granted medRxiv a license to display the preprint in perpetuity.

It is made available under a CC-BY-ND 4.0 International license .

\begin{tabular}{|c|c|c|c|c|c|c|}
\hline 21. & Connections & $\begin{array}{l}\text { National Institute } \\
\text { on Aging }\end{array}$ & $2002-2010$ & Online & Unknown & \\
\hline 22. & $\begin{array}{l}\text { Contemporary } \\
\text { Gerontology } \\
1069-0840\end{array}$ & $\begin{array}{l}\text { Springer Publishing } \\
\text { Company }\end{array}$ & 2005 & Both & Unknown & \\
\hline 23. & $\begin{array}{l}\text { Current Medical } \\
\text { Literature (CML) } \\
\text { Health Care of Older } \\
\text { People } \\
1366-8544\end{array}$ & $\begin{array}{l}\text { Remedica } \\
\text { Publishing }\end{array}$ & 2003 & Both & Unknown & \\
\hline 24. & $\begin{array}{l}\text { Ethics, Law and } \\
\text { Aging Review } \\
1540-0948\end{array}$ & $\begin{array}{l}\text { Springer Publishing } \\
\text { Company }\end{array}$ & $1995-2005$ & Both & No & \\
\hline 25. & $\begin{array}{l}\text { Geriatric Mental } \\
\underline{\text { Health Care }} \\
2212-9693\end{array}$ & Elsevier & 2013-2015 & Online & No & 0.4 \\
\hline 26. & $\begin{array}{l}\text { Geriatric Nephrology } \\
\text { and Urology } \\
\text { 0924-8455; } \\
\text { 1573-7306 }\end{array}$ & $\begin{array}{l}\text { Springer Publishing } \\
\text { Company }\end{array}$ & 1992-1999 & Both & Unknown & \\
\hline 27. & $\begin{array}{l}\text { Geriatrics (Absorbed } \\
\text { into Patient Care) } \\
\text { 0016-867X; } \\
\text { 1936-5764 }\end{array}$ & $\begin{array}{l}\text { Advanstar } \\
\text { Communications } \\
\text { Incorporated }\end{array}$ & $1946-2009$ & Both & Unknown & \\
\hline 28. & $\begin{array}{l}\text { Geriatrics \& Aging } \\
1488-8408 ; \\
1488-8416\end{array}$ & $\begin{array}{l}\text { Medscape } \\
\text { Education }\end{array}$ & $1998-2010$ & Both & Unknown & \\
\hline 29. & $\frac{\text { Geriaction }}{1032-4410}$ & $\begin{array}{l}\text { Chronic and } \\
\text { Geriatric Nursing } \\
\text { Group }\end{array}$ & 1970-2009 & Both & & \\
\hline 30. & $\begin{array}{l}\frac{\text { GeroFam }}{1309-6478 ;} \\
1309-8675\end{array}$ & $\begin{array}{l}\text { University of } \\
\text { Akdeniz }\end{array}$ & 2010-2012 & Both & Unknown & \\
\hline 31. & $\begin{array}{l}\text { Growth, Development } \\
\text { and Aging } \\
1041-1232\end{array}$ & $\begin{array}{l}\text { Growth Publication } \\
\text { Company }\end{array}$ & $1988-2008$ & Print & No & \\
\hline 32. & Hallym International & Baywood & 1999-2010 & Both & Unknown & \\
\hline
\end{tabular}


medRxiv preprint doi: https://doi.org/10.1101/19012278; this version posted November 22, 2019. The copyright holder for this preprint (which was not certified by peer review) is the author/funder, who has granted medRxiv a license to display the preprint in perpetuity.

It is made available under a CC-BY-ND 4.0 International license .

\begin{tabular}{|c|c|c|c|c|c|c|c|}
\hline & $\begin{array}{l}\text { Journal of Aging } \\
\text { 1535-6523; } \\
1541-4485\end{array}$ & $\begin{array}{l}\text { Publishing } \\
\text { Company }\end{array}$ & & & & & \\
\hline 33. & $\begin{array}{l}\text { Healthy Aging \& } \\
\text { Clinical Care in the } \\
\text { Elderly } \\
1179-0601\end{array}$ & SAGE Publications & 2008-2017 & Online & Yes & & \\
\hline 34. & $\begin{array}{l}\text { International Journal } \\
\text { on Disability and } \\
\text { Human Development } \\
2191-0367\end{array}$ & De Gruyter & $2000-2017$ & Online & No & 0.16 & \\
\hline 35. & $\frac{\text { ISRN Geriatrics }}{2314-4750}$ & Hindawi Limited & 2013-2014 & Both & Yes & & \\
\hline 36. & $\begin{array}{l}\text { Journal of Aging, } \\
\text { Humanities, and the } \\
\underline{\text { Arts }} \\
\text { 1932-5614; } \\
1932-5622\end{array}$ & $\begin{array}{l}\text { Taylor \& Francis } \\
\text { Online }\end{array}$ & 2007-2010 & Both & Optional & & \\
\hline 37. & $\begin{array}{l}\text { Journal of Aging and } \\
\text { Pharmacotherapy } \\
\text { 1540-5303; } \\
1540-4706\end{array}$ & $\begin{array}{l}\text { Taylor \& Francis } \\
\text { Online }\end{array}$ & $1995-2006$ & Online & Unknown & & \\
\hline 38. & $\begin{array}{l}\text { Journal of Geriatrics } \\
2356-7414 ; \\
2314-7121\end{array}$ & Hindawi Limited & 2014-2016 & Online & Yes & & \\
\hline 39. & $\begin{array}{l}\text { Journal of } \\
\text { Neurodegeneration \& } \\
\underline{\text { Regeneration }} \\
1932-1481\end{array}$ & $\begin{array}{l}\text { Weston Medical } \\
\text { Publishing }\end{array}$ & $2008-2013$ & Print & Unknown & & \\
\hline 40. & $\begin{array}{l}\text { Longevity \& } \\
\text { Healthspan } \\
2046-2395 \\
\end{array}$ & $\begin{array}{l}\text { Springer Publishing } \\
\text { Company }\end{array}$ & 2012-2014 & Online & Yes & & \\
\hline 41. & $\begin{array}{l}\text { Neuroembryology } \\
\text { and Aging } \\
1661-3406 ; \\
1661-3414\end{array}$ & Karger & $2002-2008$ & Both & Unknown & & \\
\hline 42. & Open Geriatric & Bentham Open & 2008-2014 & Online & Yes & & Yes (Beall's \\
\hline
\end{tabular}


medRxiv preprint doi: https://doi.org/10.1101/19012278; this version posted November 22, 2019. The copyright holder for this preprint (which was not certified by peer review) is the author/funder, who has granted medRxiv a license to display the preprint in perpetuity.

It is made available under a CC-BY-ND 4.0 International license.

\begin{tabular}{|c|c|c|c|c|c|c|c|}
\hline & $\begin{array}{l}\text { Medicine Journal, The } \\
\text { 1874-8279 }\end{array}$ & & & & & & List) \\
\hline 43. & $\begin{array}{l}\text { Open Longevity } \\
\text { Science } \\
\text { 1876-326X }\end{array}$ & Bentham Open & 2007-2013 & Online & Yes & 0 & $\begin{array}{l}\text { Yes (Beall's } \\
\text { List) }\end{array}$ \\
\hline 44. & $\begin{array}{l}\text { Reviews in Clinical } \\
\text { Gerontology } \\
\text { 0959-2598; } \\
\text { 1469-9036 }\end{array}$ & $\begin{array}{l}\text { Cambridge } \\
\text { University Press }\end{array}$ & 1991-2015 & Both & No & 0.48 & \\
\hline 45. & $\begin{array}{l}\underline{\text { Revista }} \\
\text { Multidisciplinar De } \\
\underline{\text { Gerontologia }} \\
\underline{1139-0921}\end{array}$ & $\begin{array}{l}\text { Nexus Medica } \\
\text { Editores }\end{array}$ & $\begin{array}{l}\text { 1998-2011; } \\
2017 \\
\text { (Magazine) }\end{array}$ & Online & Mixed & & \\
\hline
\end{tabular}

\section{References}

1. SJR: Scimago Journal \& Country Rank. Available online: https://www.scimagojr.com/ (accessed on 21 December 2018).

2. Clarivate Analytics. Available online: https://clarivate.com/ (accessed on 21 December 2018).

3. JenAge Information Centre. Available online: http://infocentre.jenage.de/ageing/journals.html (accessed on 21 December 2018).

4. PubMed.gov. Available online: https://www.ncbi.nlm.nih.gov/pubmed?holding=nlmlib (accessed on 21 December 2018).

5. Beall's List of Predatory Journals and Publishers. Available online: https://beallslist.weebly.com/ (accessed on 26 September 2018).

6. Beall, J. What I learned from predatory publishers. Biochem Med 2017, 27(2), 273-8, 10.11613/BM.2017.029.

7. Ferris, L.E.; Winker, M.A. Ethical issues in publishing in predatory journals. Biochem Med 2017, 27(2), 279-84, 10.11613/BM.2017.030.

8. Johal, J; Ward, R.; Gielecki, J.; Walocha, J.; Natsis, K.; Tubbs, R.S.; Loukas, M. Beware of the Predatory Science Journal: A Potential Threat to the Integrity of Medical Research. Clin Anat 2017, 30(6), 767-773, 10.1002/ca.22899.

9. Bradley-Springer, L. Predatory Publishing and You. J Assoc Nurses AIDS Care 2015, 26(3), 219-221, 10.1016/j.jana.2015.02.003. 
medRxiv preprint doi: https://doi.org/10.1101/19012278; this version posted November 22, 2019. The copyright holder for this preprint (which was not certified by peer review) is the author/funder, who has granted medRxiv a license to display the preprint in perpetuity.

It is made available under a CC-BY-ND 4.0 International license .

10. Hunziker, R. Avoiding Predatory Publishers in the Post-Beall World: Tips for Writers and Editors. AMWA Journal 2017, 32(3), 113-115.

11. Shamseer, L.; Moher, D.; Maduekwe, O.; Turner, L.; Barbour, V.; Burch, R.; Clark, J.;

Galipeau, J.; Roberts, J.; Shea, B.J. Potential predatory and legitimate biomedical journals: can you tell the difference? A cross-sectional comparison. BMC Medicine 2017, 15(28), 10.1186/s12916-017-0785-9.

12. Swauger, S. Open access, power, and privilege: A response to "What I learned from predatory publishing." CRL News 2017, 78(11), 603.

13. Teixeira da Silva, J.A. Caution with the continued use of Jeffrey Beall's "predatory" open access publishing lists. AME MED J, 2017, 2(97), 10.21037/amj.2017.06.14. 